\title{
Use of medicines in nursing homes for older people
}

\author{
Lee Furniss
}

The National Health Service (NHS) spent $£ 10$ billion (40\%) of its total budget on people aged 65 and over in 1998/1999. The profile of the health and social care of older people has been raised recently by the publication of the National Service Framework (NSF) for Older People (Department of Health, 2001). The NSF contains standards that older people can expect when they receive health and social care (Box 1). The document also discusses in detail medication management issues in older people. Its two aims in this area are to ensure that older people gain the maximum benefit from their medication in order to maintain or improve quality and duration of life, and do not suffer unnecessarily from illness caused by excessive, inappropriate or inadequate consumption of medicines.

In this paper I focus on older people who are cared for in nursing homes - arguably the most vulnerable group. I concentrate on the evidence available concerning medicine use in this population, with emphasis on psychotropic medication. I also explore the benefits of multi-disciplinary working in this area, in particular the useful contribution that pharmacists can make to the care of these patients.

\section{Medicating an ageing population}

The number of people in England expected to live into their ninth decade is expected to double over the next 20 years (Department of Health, 2001). A population-based study has predicted a large increase in the potential number of people in
England, Scotland and Wales requiring nursing and residential care in the future (Meltzer et al, 1997). Also, the levels of physical and mental ill health in residents entering homes are increasing (Stern et al, 1993). Older nursing home residents receive up to four times as many prescription items as older people living in their own homes (Walley \& Scott, 1995). The NSF states that $80 \%$ of people over the age of 75 take at least one medicine and it expects these patients to receive an annual medication review. Just over a third of people over 75 years take four or more medicines, and for these patients the NSF recommends a 6-monthly medication review.

In Ireland, residents of nursing homes are prescribed a mean of four medicines (range 0-14); almost half take five medicines or more. In the USA, the mean is 6.5 medicines per resident and the figure is similar in England. Some residents here receive as many as 17 drugs (Furniss et al, 2000).

Older people are particularly sensitive to the effects of medication and iatrogenic disease is often

Box 1 The eight standards of care in the National Service Framework for Older People (Department of Health, 2001)

1 Rooting out age discrimination

2 Person-centred care

3 Intermediate care

4 General hospital care

5 Stroke

6 Falls

7 Mental health in older people

8 Promoting an active and healthy life

Lee Furniss is Chief Pharmacist for Camden and Islington Community Health Services NHS Trust (St Luke's Hospital, Woodside Avenue, London N10 3HU, UK). The trust provides a pharmacy service to Camden and Islington Mental Health Services NHS Trust. His research interests include medication review in nursing and residential homes, primary care prescribing issues and the effective utilisation of pharmacists in multi-disciplinary teams. 
a cause of hospital admission. In the UK, studies have shown that up to one-fifth of admissions of older people to hospital may be due to inappropriate drug therapy and that up to half of these admissions may be preventable (Lindley et al, 1992).

As nursing home residents are taking the most drugs, they are particularly at risk of harm from the medicines that they take. A recent study looked at the incidence and preventability of adverse drug events in US nursing homes (Gurwitz et al, 2000). It showed that actual and potential adverse events occurred at a rate of 2.5 per 100 resident-months, with half of these judged to be preventable. Errors resulting in preventable adverse events occurred most often at the stage of ordering and monitoring of medicines. Numbers of medicines prescribed may be a predictor of death within 4 weeks of admission to a nursing home (Dale et al, 2001).

\section{Psychotropic medication}

Psychotropic drugs were widely prescribed in the USA until the Nursing Home Reform Amendments. These amendments were a component of the Omnibus Reconciliation Act (OBRA) 1987 and came into effect in October 1990. They were the result of increasing public concern about the overuse of antipsychotics in nursing homes. At the time, up to $74 \%$ of residents were receiving centrally acting medicines, which were often administered for 6 months or more (Buck, 1988). Many of these residents did not have a documented mental illness. For example, a study of 526 nursing home residents found that $21 \%$ of those receiving psychotropic medication did not have a psychiatric or organic brain disorder. OBRA recommendations explicitly state that antipsychotics should be prescribed only if psychotic phenomena exist and not for indications such as wandering, anxiety and insomnia.

In England, Lunn et al (1997) describe $26 \%$ of their population to be using 'central nervous system' drugs as defined by the British National Formulary (BNF; British Medical Association \& Royal Pharmaceutical Society of Great Britain, 2002), but this definition includes anti-epileptics and analgesics. In 1995, Snowdon et al reported that psychotropic drug use in Australian nursing homes was 59\%, although this figure has fallen in recent years (Snowdon, 1999).

\section{Antipsychotics}

In the USA, the Nursing Home Reform Amendments saw antipsychotic prescribing fall by a third, and use has since been reported to vary between $20 \%$ and 50\% (Shorr et al, 1994). The amendments limit the use of antipsychotic medication to specific clinical conditions and demand regular medication review and documentation of decisions. Homes are fined if they fail to adhere to the amendments.

In Ireland, 19\% of older people in nursing homes were reported to be taking phenothiazines (Panmore et al, 1995). This figure will probably be lower now, following a tightening recently of the licensed indications for thioridazine. In the UK, a study of south Manchester nursing homes showed that 30\% of residents were taking antipsychotics (Furniss et al, 2000).

Two studies have looked at the appropriateness of antipsychotic prescribing in the UK. In Scotland, where antipsychotic use in nursing homes was reported to be $24 \%$, McGrath \& Jackson (1996) found that $88 \%$ of these prescriptions were inappropriate if the US criteria for use was applied. This study relied solely on the use of written records to define appropriateness. In our study (Furniss et al, 2000) my colleagues and I interviewed staff and found a lower figure of $54 \%$ of antipsychotics' prescriptions inappropriate according to the US criteria. Recent work from Denmark has suggested that behavioural problems were a determinant for the use of antipsychotics (and benzodiazepines), irrespective of the psychiatric diagnosis of the resident (Sorensen et al, 2001). The authors conclude that staff perceptions of psychiatric morbidity have a greater impact on the prescription of psychotropics than do standardised clinical criteria.

\section{Atypical antipsychotics}

At the time of writing, none of the atypical drugs (as defined in the BNF) are licensed for the treatment of agitation in the older person and antipsychotics should be used cautiously (if at all) for this indication. These newer drugs have a different sideeffect profile than the typical ones. They cause fewer movement disorders, but may cause comparatively more of other side-effects such as drowsiness. For example, a study comparing the use of olanzapine with placebo showed that somnolence and gait disturbances were more common with the active treatment, which may lead to increased falls and failure to meet standard 6 of the NSF (Street et al, 2000). However, the study was only placebocontrolled, so it could not determine how the drug compares with typical agents in this respect.

\section{Antidepressants}

The prevalence of depression in nursing homes varies between $12 \%$ and $32 \%$ (Heston et al, 1992). 
Given its high prevalence and serious consequences, appropriate identification and treatment of depression must be a priority for health professionals. Antidepressants have been reported to be underused in nursing homes, their rate of use being between 6\% and $15 \%$ (Harrington et al, 1992). However, this figure has increased to $25 \%$ in recent years (Furniss et al, 2000).

\section{Hypnotics}

A number of studies (e.g. Furniss et al, 2000) over the past decade have found that $25-30 \%$ of private nursing home residents regularly take hypnotics. Significantly fewer patients in hospital continuing care wards take these medicines. Traditionally when benzodiazepines have been used, those with a shorter half-life have been recommended. However, recent work has shown that although the risk of falls among patients taking such medicines is less than for the long-acting agents, all benzodiazepines are associated with a materially increased risk of nocturnal falls (Ray et al, 2000). If benzodiazepines are to be withdrawn, this should be done gradually and residents may need greater staff involvement during this time.

\section{Unwanted effects of psychotropics}

Regular use of psychotropic medication is associated with an increased risk of recurrent falls (Thapa et al, 1995) and of fractures in the neck of the femur (Lauritzen et al, 1993). Another study used case-control methods to explore the relationship between falls, drugs and diagnosis in elderly residents of a nursing home (Granek et al, 1987). It found that those taking sedatives and antidepressants were at a greater risk of falling. Medication changes are particularly important in this respect: a change during the previous 2 weeks is a significant risk factor for falling.

The beneficial effects of antipsychotic drugs must be balanced against extrapyramidal and other (e.g. constipation, drowsiness) side-effects. Long-term use is associated with tardive dyskinesia. Studies have found associations between the use of antipsychotic drugs and restlessness, wandering, falls and incontinence of urine (Granek et al, 1987; Nygaard et al, 1990). Antipsychotic drug use may be associated with an increased rate of cognitive decline in dementia (McShane et al, 1997).

\section{Educational programmes}

Although regulations in the USA impose penalties for inappropriate use of antipsychotics in nursing homes, guidance is needed generally on how to address behavioural problems. It is useful here to identify two studies that have looked at the provision of educational programmes to medical and nursing staff employed in nursing homes and the effects of such programmes on prescribing (Avorn et al, 1992; Ray et al, 1993). Drug use and clinical status were used as outcome measures. Both studies showed a reduction in the use of antipsychotic drugs with no deterioration in functioning of residents. Neither study showed an increase in disruptive behaviour, but one showed an increase in the level of depression in the group with the greatest drug reduction. This could imply that the sedative effects of psychotropic drugs were masking depressive symptoms or that antidepressants were inappropriately stopped in some instances. However, another study has shown an improvement in the affect of residents whose antipsychotics were discontinued, which suggests that the former explanation is more likely (Thapa et al, 1994). The education of physicians alone showed no effect on the prescription of antipsychotic medication. Together these three studies imply the need to involve all the staff in a nursing home in any educational programmes.

\section{Non-psychotropic medication}

The most commonly prescribed non-psychotropic drugs in nursing homes in the UK include diuretics, analgesics, potassium salts, cardiac glycosides and laxatives; the three most commonly prescribed classes are central nervous system, cardiovascular and gastrointestinal drugs. These seem to be broadly consistent findings across a number of studies. The rate of prescribing of medicines from a recent UK study (Furness et al, 2000) is shown in Table 1.

In US nursing homes, up to $40 \%$ of residents had at least one inappropriate medication prescribed (Beers et al, 1992). In a study carried out in England, a physician (in elderly care), a general practitioner (GP), a pharmacist and a clinical pharmacologist developed criteria for inappropriate prescribing and these were then applied to the medication regimes of 101 residents (Beers et al, 1992). It was found that $53 \%$ of residents had at least one inappropriate prescription, with cardiovascular and central nervous system drugs being implicated most often (Lunn et al, 1997).

Examples of inappropriate use of non-psychotropic medicines in nursing home residents are numerous. These include the preferred use of non-steroidal anti-inflammatory drugs such as 
Table 1 Most common drug classes

prescribed to nursing home residents

Drug type

$\%$ of residents

Laxatives

49

Diuretics

Antipsychotics

44

Hypnotics/anxiolytics

Anti-platelet

Antidepressants

Analgesics

Ulcer-healing

Musculoskeletal

Nitrates/calcium channel blockers

Anti-Parkinsonian

diclofenac instead of paracetamol-based regimes in residents with osteo-arthritis and the underuse of angiotensin-converting-enzyme inhibitors such as enalapril in patients with heart failure, to prevent congestive disease.

\section{Benefits of effective medication management}

USA

In the USA, nursing homes are required by law to employ a consultant pharmacist to review prescribed medication every 1-3 months. They advise medical practitioners on matters such as choice, duration and monitoring of drug therapy and also participate in staff education about medication. Physician acceptance of pharmacist recommendations is high and has been reported to be between $60 \%$ and $81 \%$ (Dyer et al, 1984).

In one study, clinical pharmacists assumed total responsibility for the drug management of a group of elderly residents in a nursing home; this group was then compared with a control group who received traditional care (Thompson et al, 1984). The pharmacists' group had improved mortality and morbidity and took approximately two fewer drugs. A potential saving of about $\$ 70000$ per year per 100 residents was identified. Other studies (e.g. Harrison et al, 1998) have shown similar economic benefits and similar reductions in the number of drugs used per patient and also demonstrate the usefulness of the pharmacist in the eradication of unnecessary and potentially harmful medication use. Drawbacks of these studies are that most are not controlled and they do not measure objective resident outcomes.

\section{UK and Europe}

In the UK, the pharmacist's role is primarily one of supply and the provision of basic advice to the nursing home about documentation and storage. A more comprehensive clinical service is rarely provided. The situation is similar throughout northern Europe, but it is beginning to change. The targets given in the NSF for Older People regarding individualised medication review (with patient and carer) require that this clinical input be increased (Department of Health, 2001).

One study has shown that 1 hour per week of a pharmacist's time can make a significant contribution to patient care in nursing homes. It found that this input was well received by nursing staff and prescribers and that GPs accepted the pharmacist's advice in $78 \%$ of cases (Lapsley, 1998). Physician acceptance was higher (91\%) in south Manchester, where $55 \%$ of interventions resulted in actual treatment modifications. In Northamptonshire, community pharmacists analysed prescriptions of nursing home residents and provided prescribing advice to the GPs. The GPs agreed with the advice in $73 \%$ of cases and it was estimated that pharmacist involvement could give a $14 \%$ reduction in the cost of prescribing.

More recent work has used randomised controlled trials (RCTs) and objective resident outcome measures to strengthen the earlier evidence base. An RCT in 33 nursing homes in Sweden demonstrated that multi-disciplinary meetings (doctor, pharmacist and nurse) reduced prescribing of psychotropic drugs and prevented polypharmacy and therapeutic duplication (Schmidt et al, 1998). Similar work in England in 14 nursing homes showed that a brief medication review reduced the amount of medication overall with no detriment to the mental and physical functioning of the patients (Furniss et al, 2000). It also showed a reduction in the use of primary and secondary care resources by the pharmacist medication review group. Structured assessment and review of residents, educational interventions and greater multi-disciplinary working have been suggested as ways of improving the care of residents in homes (Hughes et al, 1999). Prescribing advice from pharmacists must be supplied on a continual basis if reductions in medication are to be maintained in the nursing home. Although the ideal frequency has not been determined, if the NSF is followed then most residents should be receiving a 6-monthly review.

The recommendations suggested by pharmacists include stopping and starting medicines, generic substitution, changes to another medicine, dose modification, changes in administration frequency, 
Box 2 Reasons for recommendations made by pharmacists to general practitioners

No indication for medication Indication present but no medication prescribed Side-effect or adverse drug reaction present Drug interaction

Contraindication

Alternative medication available

Safer/more efficacious use of medication Therapeutic duplication of medication Review of treatment for individual drugs Rationalisation of whole treatment Economy

formulation change and requests for laboratory tests or nurse monitoring. Examples of reasons for such recommendations are given in Box 2. In our study, my colleagues and I found that almost half of all recommendations were to stop medication and twothirds of these were because there was no indication for the drug prescribed (Furniss et al, 2000). This suggests that medication regimes are not reviewed thoroughly. Conversely, initiation of medication made up $8 \%$ of recommendations, which implies that indications are present but not always treated. This might have been due to age discrimination, which is not to be tolerated since the publication of the NSF for Older People. Fifteen per cent of recommendations were for laboratory test requests (e.g. blood profiles or therapeutic drug monitoring), suggesting that laboratory test monitoring of drug therapy is not often carried out in nursing homes. It can be seen that the majority of interventions were to improve patient care, but a small number, such as generic prescribing, were for purely economic reasons.

Pharmacists offering specialist clinical services to nursing homes will often have received postgraduate training in therapeutics, usually to Masters degree level, although many interventions require only a basic knowledge of drug treatment in elderly people. Health authorities may enter into a contract for an advisory service with local community pharmacists or it may be purchased from local hospital pharmacy departments, thus using clinical pharmacists. A clinical pharmacist is employed on the Isle of Wight with the sole remit of reviewing medication regimes in nursing homes.

As well as issuing the repeat prescriptions for residents, GPs may themselves carry out detailed medication reviews, and this too has been shown to reduce the prescribing of inappropriate medicines (Khunti \& Kinsella, 2000). It is a requirement of the
GP contract in the UK that all patients over 75 receive an annual medication review. Given the additional recommendations within the NSF for Older People concerning 6-monthly review for people taking four or more medicines, there are significant implications here for the workload of GPs. Nursing home residents may benefit from a multi-disciplinary review of their medicines. Indeed, the GPs in our study (Furniss et al, 2000) were keen to discuss with the pharmacist the use of medication in residents. However, they often described a lack of time to carry out adequate medication reviews, both in this population and in other patients on chronic multiple-drug therapy.

\section{Conclusions}

The main findings of this review are summarised in Box 3 . As time passes, it becomes increasingly likely that members of our family (and we ourselves) will be placed in nursing or residential care. Medicines form an important and significant part of treatment packages for older people. When used and mon-

\section{Box 3 Main findings of this review}

The recently published National Service Framework (NSF) for Older People sets out the standards of care that older people can expect from health and social services.

Nursing home residents are an increasing proportion of the population of older people. They are prescribed more drugs than their counterparts living at home and are often taking inappropriate medication.

Iatrogenic disease is high in older people. This is important in nursing home residents because they are prescribed more drugs and have greater physical and mental illness. The NSF for Older People specifies that patients should receive at least an annual medication review.

US pharmacists play an active role in medication management in nursing homes and this has been shown to be cost-effective. In the UK, an expanding evidence base shows the benefit of pharmacist-led medication review in conjunction with GPs. Benefits include reduced medication, reduced use of primary and secondary care resources and improved cost-effectiveness. 
itored appropriately, medicines can relieve much morbidity and delay mortality. A multi-disciplinary approach should be adopted to improve the care of nursing home residents and help to reduce the number of drug-induced problems in this population. Pharmacists have an important role to play in multi-disciplinary health and social services teams and they must be integrated into any proposed models of care. Older people in nursing homes are a vulnerable group who deserve the same high-quality clinical care as people of any age living at home.

\section{References}

Avorn, J., Soumerai, S. B., Everitt, D. E., et al (1992) A randomised trial of a program to reduce the use of psychoactive drugs in nursing homes. New England Journal of Medicine, 327, 168-173.

Beers, M. H., Ouslander, J. G., Fingold, S. F., et al (1992) Inappropriate medication prescribing in skilled-nursing facilities. Annals of Internal Medicine, 117, 684-689.

British Medical Association \& Royal Pharmaceutical Society of Great Britain (2002) British National Formulary. London \& Wallingford: BMJ Books \& Pharmaceutical Press.

Buck, J. A. (1988) Psychotropic drug practice in nursing homes. Journal of the American Geriatrics Society, 36, 409418

Dale, M. C., Burns, A., Panter, L., et al (2001) Factors affecting survival of elderly nursing home residents. International Journal of Geriatric Psychiatry, 16, 70-76.

Department of Health (2001) National Service Framework for Older People. London: Stationery Office.

Dyer, C. C., Oles, K. S., Davis, S. W. (1984) The role of the pharmacist in a gereatric nursing home: a literature review. Drug Intelligence and Clinical Pharmacy, 18, 428-433.

Furniss, L., Burns, A., Craig, S. K. L., et al (2000) Effects of a pharmacist's medication review in nursing homes. Randomised controlled trial. British Journal of Psychiatry, 176, 563-567.

Granek, E., Baker, S. P., Abbey, H., et al (1987) Medications and diagnoses in relation to falls in a long-term care facility. Journal of the American Geriatric Society, 35, 503511

Gurwitz, J. H., Field, T. S., Avorn, J., et al (2000) Incidence and preventability of adverse drug events in nursing homes. American Journal of Medicine, 109, 87-94.

Harrington, C., Tompkins, C., Curtis, M., et al (1992) Psychotropic drug use in long-term care facilities: a review of the literature. Gerontologist, 32, 822-833.

Harrison, D. L., Bootman, J. L. \& Cox, E. R. (1998) Costeffectiveness of consultant pharmacists in managing drugrelated morbidity and mortality at nursing facilities. American Journal of Health System Pharmacists, 55, 15881594.

Heston, L. L., Garrard, J., Makris, L., et al (1992) Inadequate treatment of depressed nursing home elderly. Journal of the American Geriatrics Society, 40, 1117-1122.

Hughes, C. M., Lapane, K. L. \& Mor, V. (1999) Impact of legislation on nursing home care in the United States: lessons for the United Kingdom. BMJ, 319, 10601063.

Khunti, K. \& Kinsella, B. (2000) Effect of systematic review of medication by general practitioner on drug consumption among nursing home residents. Age and Ageing, 29, 451453.

Lapsley, R. (1998) Prescription monitoring in a nursing home. Pharmaceutical Journal, 240, 688-690.
Lauritzen, J., McNair, P. \& Lund, B. (1993) Risk factors for hip fractures. Danish Medical Bulletin, 40, 479-485.

Lindley, C. M., Tully, M. P., Paramsothy, V., et al (1992) Inappropriate medication is a major cause of adverse drug reactions in elderly patients. Age and Ageing, 21, 294-300.

Lunn, J., Chan, K. \& Donoghue, J. (1997) A study of the appropriateness of prescribing in nursing homes. International Journal of Pharmaceutical Practice, 5, 6-10.

McGrath, A. M. \& Jackson, G. A. (1996) Survey of neuroleptic prescribing in residents of nursing homes in Glasgow. BMJ, 312, 611-612.

McShane, R., Keene, J., Gedling, K., et al (1997) Do neuroleptic drugs hasten cognitive decline in dementia? Prospective study with necropsy follow up. BMJ, 314, 266-270.

Meltzer, D., Ely, M. \& Brayne, C. (1997) Cognitive impairment in elderly people: population based estimate of the future in England, Scotland and Wales. $B M J, 315,462$.

Nygaard, H. A., Bakke, K. J., Breivik, K., et al (1990) Mental and physical capacity and consumption of neuroleptic drugs in residents of nursing homes. International Journal of Geriatric Psychiatry, 5, 303-308.

Panmore, A. P., Crawford, V. L. S., Beringer, T. R. O., et al (1995) Determinants of drug utilisation in an elderly population in north and west Belfast. Parmacoepidemiology and Drug Safety, 4, 147-160.

Ray, W. A., Taylor, J. A., Meador, K. G., et al (1993) Reducing antipsychotic drug use in nursing homes. Archives of Internal Medicine, 15, 713-721.

- Thapa, P. B. \& Gideon, P. (2000) Benzodiazepines and the risk of falls in nursing home residents. Journal of the American Geriatrics Society, 48, 682-685.

Schmidt, I., Claesson, C. B., Westerholm, B., et al (1998) The impact of regular multidisciplinary team interventions on psychotropic prescribing in Swedish nursing homes. Journal of the American Geriatrics Society, 46, 4782

Shorr, R. I., Fought, R. L. \& Ray, W. A. (1994) Changes in antipsychotic drug use in nursing homes during implementation of the OBRA-87 regulations. JAMA, 271, $358-362$

Snowdon, J. (1999) A follow-up survey of psychotropic drug use in Sydney nursing homes. Medical Journal of Australia, 170, 299-301.

—, Vaughan, R., Miller, R., et al (1995) Psychotropic drug use in Sydney nursing homes. Medical Journal of Australia, $163,70-72$.

Sorensen, L., Foldspang, A., Gulmann, N. C., et al (2001) Determinants for the use of psychotropics among nursing home residents. International Journal of Geriatric Psychiatry, 16, 147-154

Stern, M. C., Jagger, C., Clark, M., et al (1993) Residential care for elderly people: a decade of change. BMJ, 306, $827-830$

Street, J. S., Clark, W. S., Gannon, K. S., et al (2000) Olanzapine treatment of psychotic and behavioural symptoms in patients with Alzheimer disease in nursing care facilities: a double-blind, randomised, placebo controlled trial. The HGEU study group. Archives of General Psychiatry, 57, 968976

Thapa, P. B., Meador, K. G., Gideon, P., et al (1994) Effects of antipsychotic withdrawal in elderly nursing home residents. Journal of the American Geriatric Society, 42, 280206.

—, Gideon, P., Fought, R. L., et al (1995) Psychotropic drugs and the risk of recurrent falls in ambulatory nursing home residents. American Journal of Epidemiology, 142, 202-211.

Thompson, J. F., McGhan, W. F., Ruffalo, R., et al (1984) Clinical pharmacists prescribing drug therapy in a geriatric setting: outcome of a trial. Journal of the American Geriatrics Society, 32, 154-159.

Walley, T. \& Scott, A. K. (1995) Prescribing in the elderly. Postgraduate Medical Journal, 71, 466-471. 


\section{Multiple choice questions}

1. The percentage of the NHS budget spent on people over 65 years of age in 1998/1999 was:
a $10 \%$
b $25 \%$
c $40 \%$
d $50 \%$
e $55 \%$.

2. Studies report the percentage of residents in UK nursing homes taking antipsychotics to be about:
a $10 \%$
b $15 \%$
c $30 \%$
d $54 \%$
e $88 \%$.

3. Atypical antipsychotics:

a should be used to treat all agitated residents in nursing homes

b cause more extrapyramidal side-effects than most typical antipsychotics

c might cause somnolence

d might cause gait disturbances

e include flupentixol and pimozide.
4. The following drugs are used relatively commonly (by more than $10 \%$ of residents) in nursing homes:
a laxatives
b oestrogens
c diuretics
d aspirin
e leukotriene receptor antagonists.

5. Studies report the percentage of residents in UK nursing homes taking antidepressants to be about:
a $10 \%$
b $25 \%$
c $35 \%$
d $40 \%$
e $45 \%$.

\section{MCQ answers}

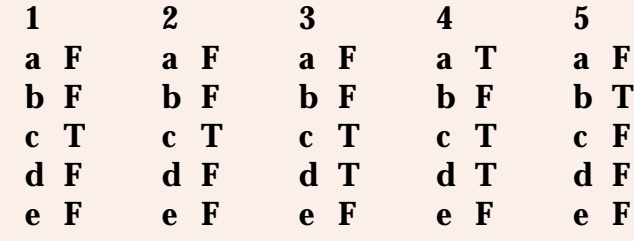

